\title{
UNSTEADY FLOW THROUGH A GASGADE OF AEROFOILS
}

\author{
A. H. LOW and L. C. WOODS \\ (received 20 August 1959)
}

\begin{abstract}
Summary
This paper extends some earlier work by Woods [8] on the transient forces caused by unsteady transverse motions of a rigid cascade of aerofoils to the case when the inlet velocity is varying in magnitude. The flow is incompressible and two-dimensional. Expressions for the growth of lift and moment on a member of the cascade with increasing inlet velocity are evaluated and shown to be generalizations of Wagner's classical results for an isolated aerofoil.
\end{abstract}

\section{Introduction}

Suppose that an unstaggered cascade of thin aerofoils or blades is set at a small incidence $\alpha$ to an inlet stream of incompressible fluid and that the velocity of this stream is increased or decreased in magnitude. One result will be a corresponding variation in the pressure distribution over a typical blade. It is this variation and the consequent variation in the force and moment acting on the blade that we shall calculate in this paper.

This type of unsteady flow is always present to some extent under normal operating conditions in turbo-machinery, for each ring of rotor blades must move through the wake (depressions in velocity magnitude) of the blades of the upstream stator. The induced fluctuating loads on each blade, even if small, will contribute to the fatigue and eventual failure of the blade.

More serious are the extreme fluctuations that may occur through turbo-machinery, e.g. the surges that arise in some compressors under abnormal operating conditions.

Woods [8] studied the loads produced on cascade blades by transverse unsteady motions of the cascade as a rigid group, or equivalently due to small changes in the inlet angle $\alpha$. This work is extended here to allow for variations in the magnitude $U$ of the inlet velocity; the earlier work is thus a special case of that presented below.

A generalisation is found for Wagner's growth of lift function. This 
generalisation lends itself to series expansions in terms of the chord: gap ratio of the cascade. The special case of constant acceleration of the cascade (relative to the inlet fluid) also receives some attention.

\section{General Theory}

As we shall restrict attention to the problem of finding the induced loading on an unstaggered cascade of thin aerofoils, distance $h$ apart, we can employ the usual linearization of boundary shape and apply the boundary conditions on the family of slits $-\frac{1}{2} c<x<\frac{1}{2} c, y= \pm n h, n=0,1,2 \ldots$
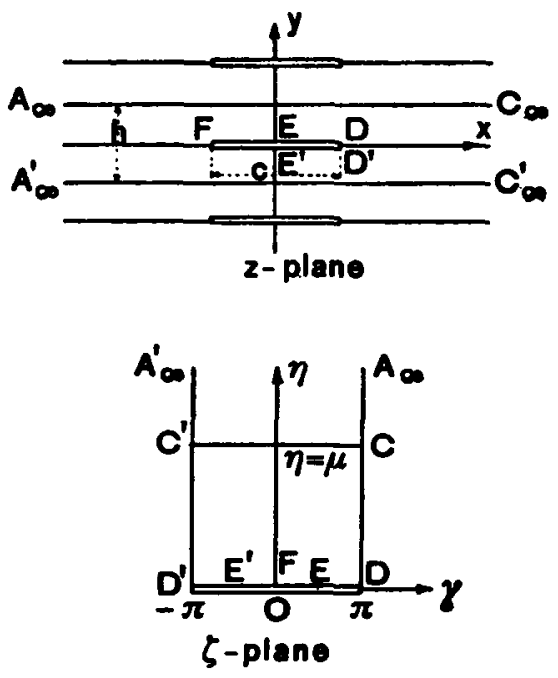

Figure 1

It is easily verified that these slits are mapped on to $-\pi<\gamma<\pi, \eta=0$ in the $\zeta$-plane $(\zeta=\gamma+i \eta)$ shown in figure 1 by

$$
\cosh r+\sinh r \cos \zeta=\exp -\left(\frac{2 r z}{c}\right) \text {. }
$$

where

$$
r=\frac{\pi c}{h}
$$

The point upstream at infinity, i.e. $x=-\infty$, maps onto $\eta=\infty$, while the points $x=\infty, y>0$ and $x=\infty, y<0$ map onto $\zeta=\pi+i \mu$ and $\zeta=-\pi+i \mu$ respectively, where from (1)

$$
\cosh \mu=\operatorname{coth} r \text {. }
$$

The upper. and lower surfaces of the vortex sheet extending behind each aerofoil of the cascade map onto $\gamma=\pi, 0 \leqq \eta<\mu$, and $\gamma=-\pi$, $0 \leqq \eta<\mu$ respectively. On the surface of an aerofoil of the cascade (1) becomes 


$$
\cosh r+\sinh r \cos \gamma=\exp -\left(\frac{2 r x}{c}\right) \text {. }
$$

The general theory developed by Woods [8] for a flow consisting of small unsteady perturbations about a steady mean flow is readily adapted to the problem now being considered, namely that of small perturbations about an unsteady mean flow. The difference in the two theories, of course, lies in the fact that, where as the inlet velocity $U$ was previously constant, in the flow now being considered $U$ is a function of time.

Let $(q, \theta)$ denote the velocity vector in polar coordinates; then the function

$$
f \equiv \ln \left\{U \frac{\mathrm{d} z}{\mathrm{~d} w}\right\}=\Omega+i \theta
$$

where $\Omega=\ln \{U / q\}$, is an analytic function of $z$ and hence of $\zeta$. As one side of the vortex sheet becomes the left-hand side of $\zeta=\pi+i \eta$ while the other becomes the right-hand side of $\zeta=-\pi+i \eta$, where $0 \leqq \eta \leqq \mu$, the jump in $\Omega$ across the vortex sheet may be written

$$
\bar{\omega} \equiv \Omega(\pi+i \eta-0)-\Omega(-\pi+i \eta+0) .
$$

The solution of the boundary value problem in which

(a) $\theta$ is known on $\eta=0,-\pi \leqq \gamma \leqq \pi$, and

(b) $\bar{\omega}$ is known on $\gamma= \pm \pi, 0<\eta<\infty$, is given by [7]

$$
f(\zeta)=\frac{1}{2 \pi} \int_{-\pi}^{\pi} \theta\left(\gamma^{*}\right) \cot \left(\frac{\gamma^{*}-\zeta}{2}\right) \mathrm{d} \gamma^{*}+\frac{\sin \zeta}{2 \pi} \int_{0}^{\infty} \frac{\bar{\omega}\left(\eta^{*}\right)}{\cosh \eta^{*}+\cos \zeta} \mathrm{d} \eta^{*}
$$

Assuming Joukowski's condition that the rear stagnation point remains fixed in position, we have, on the aerofoil surface (by "the" aerofoil we mean the typical aerofoil lying on $y=0$ ).

$$
\theta=\theta_{\mathrm{s}}+\frac{n}{U}-\alpha+\pi \lambda \delta(\gamma)
$$

where $\theta_{\mathrm{a}}$ is the slope of the surface, $n$ is the normal velocity of the surface, $\alpha$ is the angle of incidence of the aerofoil (measured from the negative $x$-axis), $\lambda$ is a number initially unknown and due to the small movement of the front stagnation point away from its mean position $\gamma=0$ (the value of $\lambda$ is fixed by the condition that the unsteady motion can have no influence on the flow direction, assumed parallel to $x$-axis, at infinity upstream), and $\delta(\gamma)$ is the delta function.

In fact, the last term on the right-hand side of (8) is due to the flow direction being reversed for points on the aerofoil surface between the mean stagnation point $(\gamma=0)$ and the perturbed stagnation point $(\gamma=\lambda$, say). This effect can be accounted for by a term 


$$
\pi\{H(\gamma-\lambda)-H(\gamma)\}
$$

where $H(\gamma)$ is the Heaviside unit function. As $\mathrm{d} / \mathrm{d} \gamma\{H(\gamma)\}=\delta(\gamma)$ this term can be written as $\pi \lambda \delta(\gamma)$, on the assumption that $\gamma-\lambda$ is small.

Upstream at infinity, i.e. at $\eta=\infty, \theta=0$ and it follows from (5) that $f(\gamma+i \infty)=0$. Kelvin's circulation theorem and the assumption there is no circulation before the commencement of the unsteady motion, yields the same result downstream at infinity (cf. [8]); thus $f(\gamma+i \mu)=0$. Substituting these limits in (7) we obtain

and

$$
\begin{aligned}
& \int_{-\pi}^{\pi} \theta\left(\gamma^{*}\right) \mathrm{d} \gamma^{*}+\int_{0}^{\infty} \bar{\omega}\left(\eta^{*}\right) \mathrm{d} \eta^{*}=0, \\
& \int_{-\pi}^{\pi} \frac{\theta\left(\gamma^{*}\right) \sin \gamma^{*} \mathrm{~d} \gamma^{*}}{\cosh \mu+\cos \gamma^{*}}=0,
\end{aligned}
$$

$$
\int_{-\pi}^{\pi} \frac{\theta\left(\gamma^{*}\right) \mathrm{d} \gamma^{*}}{\cosh \mu+\cos \gamma^{*}}-\int_{0}^{\infty} \frac{\bar{\omega}\left(\eta^{*}\right) \mathrm{d} \eta^{*}}{\cosh \eta^{*}-\cosh \mu}=0
$$

Let $\chi=U \bar{\omega}$, then $-\chi$ is the strength of the vorticity sheet in the aerofoil wake and, by the persistence of vorticity, satisfies

$$
U \cdot \frac{\partial \chi}{\partial x}+\frac{\partial \chi}{\partial t}=0
$$

or

$$
\frac{\partial \chi}{\partial x}+\frac{2}{c} \frac{\partial \chi}{\partial \mathrm{J}}=0
$$

where $\mathrm{J}$ is the "reduced time",

$$
\mathrm{J} \equiv \frac{2}{c} \int_{0}^{t} U \mathrm{~d} t
$$

This "time" equals the total length of the vortex sheet, in units of halfchord length, for an unsteady motion that commenced at $t=0$. The solution of $(12)$ is

$$
\chi(x, \mathrm{~J})=\chi\left(\frac{c}{2}, \mathrm{~J}+1-\frac{2 x}{c}\right)
$$

which relates $\mathrm{J}$ at any point and time to its value at the trailing edge $x=c / 2$ at an earlier time. Let

$$
\xi=\frac{2 x}{c},
$$

then (14) can be written

$$
\chi(\xi, \mathrm{J})=\chi(1, \mathrm{~J}+1-\xi) .
$$


In the next section we shall use the above expressions to obtain an explicit equation for $\chi$. Apart from the fact that $U$ is now a function of time, the calculation is similar to that given in Woods [8].

\section{The Strength of Trailing Vortex Sheets}

In the special case of steady flow at zero incidence, there is no trailing vortex sheet and equation (8) reduces to

$$
\theta=\theta_{\mathrm{s}}
$$

so that, from equations (9) and (11)

$$
\int_{-\pi}^{\pi} \theta_{B}\left(\gamma^{*}\right) \mathrm{d} \gamma^{*}=0
$$

and

$$
\int_{-\pi}^{\pi} \frac{\theta_{\mathrm{B}}\left(\gamma^{*}\right)}{\cosh \mu+\cos \gamma^{*}} \mathrm{~d} \gamma^{*}=0
$$

For the general case of unsteady flow about an aerofoil at some small incidence we have from equations (9), (10) and (11), using (8), (17) and (18)

$$
\lambda U=2 \alpha U-\frac{1}{\pi} \int_{-\pi}^{\pi} n\left(\gamma^{*}, \mathrm{~J}\right) \mathrm{d} \gamma^{*}-\frac{1}{\pi} \int_{0}^{\infty} \chi\left(\eta^{*}, \mathrm{~J}\right) \mathrm{d} \eta^{*} ;
$$

$$
0=U \int_{-\pi}^{\pi} \theta_{8}\left(\gamma^{*}\right) \frac{\sin \gamma^{*} \mathrm{~d} \gamma^{*}}{\cosh \mu+\cos \gamma^{*}}+\int_{-\pi}^{\pi} n\left(\gamma^{*}, \mathrm{~J}\right) \frac{\sin \gamma^{*}}{\cosh \mu+\cos \gamma^{*}} \mathrm{~d} \gamma^{*}
$$

ind

$$
\begin{aligned}
\frac{\lambda U}{1+\cosh \mu}=\frac{2 \alpha U}{\sinh \mu} & -\frac{1}{\pi} \int_{-\pi}^{\pi} \frac{n\left(\gamma^{*}, \mathrm{~J}\right)}{\cosh \mu+\cos \gamma^{*}} \mathrm{~d} \gamma^{*} \\
& +\frac{1}{\pi} \int_{0}^{\infty} \frac{\chi\left(\eta^{*}, \mathrm{~J}\right)}{\cosh \eta^{*}-\cosh \mu} \mathrm{d} \eta^{*} .
\end{aligned}
$$

Equation (19) fixes the value of $\lambda$; equation (20) is a (physical) restriction in the unsteady perturbations while $(21)$ is an integral equation for the rortex sheet strength $-\chi$.

Equations (19) and (21) may be rewritten, using equations (3) and (15), a the form

22)

$$
\begin{aligned}
\lambda U= & 2 \alpha U-\frac{1}{\pi} \int_{-\pi}^{\pi} n\left(\gamma^{*}, \mathrm{~J}\right) \mathrm{d} \gamma^{*} \\
& -\frac{r \mathrm{e}^{-r / 2}}{\pi \sqrt{2}} \int_{-\infty}^{\infty}[H(\nu) \chi(1, v)]\left[\frac{H(\mathrm{~J}-v) \mathrm{e}^{-r / 2(\mathrm{~J}-\nu)}}{\sqrt{\cosh r(\mathrm{~J}-\nu+1)-\cosh r}}\right] \mathrm{d} v
\end{aligned}
$$

nd 


$$
\begin{aligned}
\lambda U= & \frac{1+\cosh \mu}{\sinh \mu} 2 \alpha U-\frac{1+\cosh \mu}{\pi} \int_{-\pi}^{\pi} \frac{n\left(\gamma^{*}, \mathrm{~J}\right) \mathrm{d} \gamma^{*}}{\cosh \mu+\cos \gamma^{*}} \\
& -\frac{(1+\cosh \mu) r \sinh r \mathrm{e}^{-\frac{1}{2} r}}{\pi \sqrt{2}} \int_{-\infty}^{\infty}[H(\nu) \chi(1, \nu)] \\
& \times \frac{H(\mathrm{~J}-\nu) \mathrm{e}^{-\frac{1}{2} r(\mathrm{~J}-\nu)}}{\sqrt{\cosh r(\mathrm{~J}-\nu+1)-\cosh r}} \mathrm{~d} \nu
\end{aligned}
$$

where

$$
\nu=\mathrm{J}+1-\xi
$$

Equations (22) and (23) can be solved by the Laplace Transform method. The notations used for the transform and its inverse will be as follows [3]:

$$
\begin{array}{cl}
\mathscr{L}\{f(\mathrm{~J}), \mathrm{p}\} \equiv \mathscr{L}\{f\} \equiv \tilde{f}(\mathrm{p})=\mathrm{p} \int_{-\infty}^{\infty} \mathrm{e}^{-\mathrm{pJ}} f(\mathrm{~J}) \mathrm{d} \mathrm{J}, & a \leqq \mathscr{R}(\mathrm{p}) \leqq b \\
\mathscr{L}^{-1}\{F(\mathrm{p}), \mathrm{J}\} \equiv \frac{1}{2 \pi i} \int_{c_{0}-i \infty}^{\mathrm{c}_{0}+i \infty} \mathrm{e}^{\mathrm{Jp}} F(\mathrm{p}) \frac{\mathrm{dp}}{\mathrm{p}} & a<c_{0}<b
\end{array}
$$

where the restrictions on the real numbers $c_{0}$ and $\mathscr{R}(\mathrm{p})$ are chosen so that convergence of the integral is ensured. Use is also made of the transform rules

$$
\mathscr{L}\left\{\int_{-\infty}^{\infty} f(\tau) F(\mathrm{~J}-\tau) \mathrm{d} \tau, \mathrm{p}\right\}=\frac{1}{\mathrm{p}} \mathscr{L}\{f(\mathrm{~J}), \mathrm{p}\} \mathscr{L}\{F(\mathrm{~J}), \mathrm{p}\}
$$

and

$$
\mathscr{L}\left\{\frac{\mathrm{d}^{\mathrm{n}} F(\mathrm{~J})}{\mathrm{d}^{\mathrm{n}}}, \mathrm{p}\right\}=\mathrm{p}^{\mathrm{n}} \mathscr{L}\{F(\mathrm{~J}), \mathrm{p}\}
$$

On writing

$$
\begin{aligned}
& A(\mathrm{~J})=\lambda(\mathrm{J}) U(\mathrm{~J}), \\
& B(\mathrm{~J})=2 \alpha(\mathrm{J}) U(\mathrm{~J}), \\
& a_{0}(\mathrm{~J})=\frac{1}{\pi} \int_{-\pi}^{\pi} n\left(\gamma^{*}, \mathrm{~J}\right) \mathrm{d} \gamma^{*},
\end{aligned}
$$

and

$$
a_{1}(\mathrm{~J})=\frac{1}{\pi} \int_{-\pi}^{\pi} \frac{n\left(\gamma^{*}, \mathrm{~J}\right)}{\cosh \mu+\cos \gamma^{*}} \mathrm{~d} \gamma^{*}
$$

we find, from equations $(3),(22),(23),(25)$ and $(27)$ that

$$
\mathscr{L}\{A\}=\mathscr{L}\{B\}-\mathscr{L}\left\{a_{0}\right\}-\frac{\mathrm{e}^{p}}{\pi} Q_{\mathrm{p} /} \mathscr{L}\left\{H_{\chi}\right\}
$$

and

$$
\mathscr{L}\{A\}=\mathrm{e}^{r} \mathscr{L}\{B\}-\frac{\mathrm{e}^{r}}{\sinh r} \mathscr{L}\left\{a_{1}\right\}-\frac{\mathrm{e}^{p+r}}{\pi} Q_{(\mathrm{p}-r) /,} \mathscr{L}\left\{H_{\chi}\right\}
$$


where the argument, $\cosh r$, of the Legendre function of the second kind $Q_{m}(\cosh r)$ (see p. 319 of [6]) has been omitted.

With the result

$$
Q_{(\mathrm{p}-r) / r}(\cosh r)=\cosh r Q_{\mathrm{p} / r}(\cosh r)-\frac{r}{\mathrm{p}} \sinh r Q_{\mathrm{p} / r}^{\mathrm{1}}(\cosh r)
$$

where $Q_{\mathrm{p} / \mathrm{r}}^{1}$ is an associated Legendre function of the first order, equations (33) and (34) yield

$$
\mathscr{L}\{A\}=\mathscr{L}\{L\}-\mathscr{L}\left\{a_{0}\right\}+\bar{g} \frac{Q_{\mathrm{p} / r}}{Q_{\mathrm{p} / r}-(r / \mathrm{p}) Q_{\mathrm{p} / r}^{1}},
$$

and

$$
\mathscr{L}\{H \chi\}=-\bar{g} \frac{\pi \mathrm{e}^{-p}}{Q_{\mathrm{p} / \boldsymbol{r}}-(\boldsymbol{r} / \mathrm{p}) Q_{\mathrm{p} / \boldsymbol{r}}^{1}},
$$

where

$$
g=-\frac{\left(e^{r}-1\right) B+a_{0}-\mathrm{e}^{r} a_{1} \operatorname{cosech} r}{\mathrm{e}^{r} \sinh r}
$$

By (28), equation (36) may be written in the form

$$
\begin{aligned}
\mathscr{L}\{A\} & =\mathscr{L}\left\{A_{1}\right\} \mathscr{L}\{g\}+\mathscr{L}\left\{B-a_{0}\right\} \\
& =\frac{1}{\mathrm{p}} \mathscr{L}\left\{A_{1}\right\} \mathscr{L}\{\dot{g}\}+\mathscr{L}\left\{B-a_{0}\right\}
\end{aligned}
$$

where the dot denotes differentiation with respect to $\mathrm{J}$. Therefore, from (25), (27) and (29)

$$
A \equiv \lambda(\mathrm{J}) U(\mathrm{~J})=\int_{0}^{\mathrm{J}} A_{1}(\mathrm{~J}-\tau) \dot{g}(\tau) \mathrm{d} \tau+B-a_{0}
$$

where

$$
A_{1}(\mathrm{~J})=\frac{1}{2 \pi i} \int_{\mathrm{c}_{0}-i \infty}^{\mathrm{c}_{0}+i \infty} \frac{\mathrm{e}^{\mathrm{pJ}} Q_{\mathrm{p} / r}}{Q_{\mathrm{p} / r}-(r / \mathrm{p}) Q_{\mathrm{p} / r}^{1}} \frac{\mathrm{dp}}{\mathrm{p}} .
$$

Similarly, from (37),

$$
\chi(\mathrm{J})=\int_{0}^{\mathrm{J}} A_{2}(\mathrm{~J}-\tau) \dot{g}(\tau) \mathrm{d} \tau
$$

where

$$
A_{2}(\mathrm{~J})=\frac{i}{2} \int_{c_{0}-i \infty}^{c_{0}+i \infty} \frac{\mathrm{e}^{\mathrm{p}(\mathrm{J}-1)}}{Q_{\mathrm{p} / r}-(r / \mathrm{p}) Q_{\mathrm{p} / r}^{1}} \frac{\mathrm{dp}}{\mathrm{p}}
$$

Equations (39), (40), (41) and (42) determine the values of $\lambda$ and $\chi$. i.e. the movement of the front stagnation point and the strength of the vortex sheet respectively, in the unsteady flow. 


\section{Series Expansions of $A_{1}(\mathrm{~J})$}

In $\S 5$ it will be seen that, in order to calculate the pressure, and hence the lift and moment, on an aerofoil of the cascade, it is sufficient to be able to evaluate $A_{1}(\mathrm{~J})$. To do this, it is found convenient to expand in series the integral by which the function is defined.

Thus, from equation (40), we first rewrite $A_{1}(\mathrm{~J})$ in the form

$$
A_{1}(\mathrm{~J}, r)=\mathscr{L}^{-1}\left\{\bar{A}_{1}(\mathrm{p}, r)\right\}
$$

where

$$
\bar{A}_{1}(\mathrm{p}, r)=\frac{Q_{\mathrm{p} / r}}{Q_{\mathrm{p} / r}-(r / \mathrm{p}) Q_{\mathrm{p} / r}^{1}} .
$$

Using a result obtained by S. Rosenblat [2] for the transformation of Legendre functions of the second kind into hypergeometric functions, namely

$$
\begin{aligned}
Q_{n}(z) & =\frac{\sqrt{ } \pi}{2^{n+1}} \frac{\Gamma(n+1)}{\Gamma\left(n+\frac{3}{2}\right)}(1+z)^{-n-1}\left\{\frac{1}{2}\left(1+\sqrt{\frac{z-1}{z+1}}\right)\right\}^{-2 n-2} \\
& \times F\left(n+1, \frac{1}{2} ; n+\frac{3}{2} ;\left[\frac{\sqrt{z+1}-\sqrt{z-1}}{\sqrt{z+1}+\sqrt{z-1}}\right]^{2}\right)
\end{aligned}
$$

equation (43) becomes

$$
=\frac{\frac{\mathrm{p}}{r}\left(1-\mathrm{e}^{-2 r}\right) F\left(\frac{\mathrm{p}}{r}+1, \frac{1}{2} ; \frac{\mathrm{p}}{r}+\frac{3}{2} ; \mathrm{e}^{-2 r}\right)}{\left(2 \frac{\mathrm{p}}{r}+1\right) F\left(\frac{\mathrm{p}}{r}, \frac{1}{2} ; \frac{\mathrm{p}}{r}+\frac{1}{2} ; \mathrm{e}^{-2 r}\right)-2 \frac{\mathrm{p}}{r} \mathrm{e}^{-2 r} F\left(\frac{\mathrm{p}}{r}+1, \frac{1}{2} ; \frac{\mathrm{p}}{r}+\frac{3}{2} ; \mathrm{e}^{-2 r}\right)} .
$$

One of the Gauss relations between contiguous functions of the hypergeometric function gives

$$
c F(a-1, b ; c ; z)-(c-b) z F(a, b ; c+1 ; z)=c(1-z) F(a, b ; c ; z)
$$

which, on letting

$$
a=\frac{\mathrm{p}}{r}+1 ; \quad b=\frac{1}{2} ; \quad c=\frac{\mathrm{p}}{r}+\frac{1}{2} ; \quad z=\mathrm{e}^{-2 r},
$$

yields

$$
\begin{aligned}
\left(\frac{\mathrm{p}}{r}+\frac{1}{2}\right) F\left(\frac{\mathrm{p}}{r}, \frac{1}{2} ; \frac{\mathrm{p}}{r}+\frac{1}{2} ; \mathrm{e}^{-2 r}\right)-\frac{\mathrm{p}}{r} \mathrm{e}^{-2 r} F\left(\frac{\mathrm{p}}{r}+1, \frac{1}{2} ; \frac{\mathrm{p}}{r}+\frac{3}{2} ; \mathrm{e}^{-2 r}\right) \\
=\left(\frac{\mathrm{p}}{r}+\frac{1}{2}\right)\left(1-\mathrm{e}^{-2 r}\right) F\left(\frac{\mathrm{p}}{r}+1, \frac{1}{2} ; \frac{\mathrm{p}}{r}+\frac{1}{2} ; \mathrm{e}^{-2 r}\right)
\end{aligned}
$$


so that from $(45)$

$$
A_{1}(\mathrm{p}, r)=\frac{1}{2} \frac{\frac{\mathrm{p}}{r}}{\frac{\mathrm{p}}{r}+\frac{1}{2}} \frac{F\left(\frac{\mathrm{p}}{r}+1, \frac{1}{2} ; \frac{\mathrm{p}}{r}+\frac{3}{2} ; \mathrm{e}^{-2 r}\right)}{F\left(\frac{\mathrm{p}}{r}+1, \frac{1}{2} ; \frac{\mathrm{p}}{r}+\frac{1}{2} ; \mathrm{e}^{-2 r}\right)}
$$

From the series expansion of the hypergeometric function we have, for $r>0$, to the second order in $\mathrm{e}^{-2 r}$,

$$
\begin{aligned}
& A_{1}(\mathrm{p}, r)=\frac{1}{2} \frac{\frac{\mathrm{p}}{r}}{\left(\frac{\mathrm{p}}{r}+\frac{1}{2}\right)}\left\{1-\frac{1}{2} \frac{\left(\frac{\mathrm{p}}{r}+1\right) \mathrm{e}^{-2 r}}{\left(\frac{\mathrm{p}}{r}+\frac{1}{2}\right)\left(\frac{\mathrm{p}}{r}+\frac{3}{2}\right)}\right. \\
& \left.-\frac{\left(\frac{\mathrm{p}}{r}+1\right)\left(4\left[\frac{\mathrm{p}}{r}\right]^{2}+8\left[\frac{\mathrm{p}}{r}\right]+1\right)}{8\left(\frac{\mathrm{p}}{r}+\frac{1}{2}\right)^{2}\left(\frac{\mathrm{p}}{r}+\frac{3}{2}\right)\left(\frac{\mathrm{p}}{r}+\frac{5}{2}\right)} \mathrm{e}^{-4 r}+\cdots\right\} .
\end{aligned}
$$

Thus, using (26), and the additional transform rules [3]

$$
\mathscr{L}\left\{\mathrm{e}^{-\lambda \mathrm{J}} f(\mathrm{~J}), \mathrm{p}\right\}=\frac{\mathrm{p}}{\mathrm{p}+\lambda} \bar{f}(\mathrm{p}+\lambda) \quad(\alpha-\mathscr{R}(\lambda)<\mathscr{R}(\mathrm{p})<\beta-\mathscr{R}(\lambda))
$$

and

$$
\mathscr{L}\left\{\mathrm{J}^{\nu} H(\mathrm{~J})\right\}=\frac{\Gamma(\nu+1)}{\mathrm{p}^{\nu}} \quad(\mathscr{R}(\nu)>-1, \quad 0<\mathscr{R}(\mathrm{p})<\infty)
$$

we have

$$
\begin{aligned}
A_{1}(\mathrm{~J}, r) & =\frac{H(\mathrm{~J})}{2}\left\{\mathrm{e}^{-\frac{1}{2} r \mathrm{~J}}-\frac{1}{4}\left[\left(1-\mathrm{e}^{-r \mathrm{~J}}\right)+r \mathrm{~J}\right] \mathrm{e}^{-\frac{1}{2} r[\mathrm{~J}+4]}\right. \\
& -\frac{1}{64}\left[\left(17+6 r \mathrm{~J}-4 r^{2} \mathrm{~J}^{2}\right)-8 \mathrm{e}^{-r \mathrm{~J}}-9 \mathrm{e}^{-2 r \mathrm{~J}}\right] \mathrm{e}^{-\frac{1}{2} r(\mathrm{~J}+8)}+\cdots \cdot .
\end{aligned}
$$

Substitution of (49) in (39) allows, for $r>0$, the determination of $\lambda U$.

An alternative expansion to (49), applicable to small values of $r$, may be obtained in the following manner. Using the Laplacian integral form for the Legendre function of the second kind (see p. 319 of [6]), equation (43) is written

$$
A_{1}(\mathrm{p}, r)=\frac{\int_{0}^{\infty}\{1+\tanh r \cosh \phi\}^{-((\mathrm{p} / r)+1)} \mathrm{d} \phi}{\int_{0}^{\infty}\{1+\cosh \phi\}\{1+\tanh r \cosh \phi\}^{-((\mathrm{p} / r)+1)} \mathrm{d} \phi}
$$

Since the derivatives, of any order with respect to $r$, of $A_{1}(\mathrm{p}, r)$ are uniformly convergent at $r=0$, we may write 
(51) $\quad A_{1}(\mathrm{p}, r)=A_{1}(\mathrm{p}, 0)+r\left\{\frac{\partial}{\partial r} A_{1}(\mathrm{p}, r)\right\}_{r=0}+\frac{r^{2}}{2 !}\left\{\frac{\partial^{2}}{\partial r^{2}} A_{1}(\mathrm{p}, r)\right\}_{r=0}+\ldots$

where the order of the integration and differentiation of the integrals defining $A_{1}(\mathrm{p}, r)$ may be interchanged.

Thus, from (50)

$$
\bar{A}_{1}(\mathrm{p}, 0)=\frac{\int_{0}^{\infty} \mathrm{e}^{-\mathrm{p} \cosh \phi} \mathrm{d} \phi}{\int_{0}^{\infty} \mathrm{e}^{-\mathrm{p} \cosh \phi}(1+\cosh \phi) \mathrm{d} \phi},
$$

i.e.

$$
A_{1}(\mathrm{p}, 0)=\frac{K_{0}(\mathrm{p})}{K_{0}(\mathrm{p})+K_{1}(\mathrm{p})}
$$

where $K_{m}(\mathrm{p})$ is a modified Bessel function of the second kind (see p. 181 of $[5])$. Use of the relation

$$
K_{m+1}(\mathrm{p})-K_{m-1}(\mathrm{p})=\frac{2 m}{\mathrm{p}} K_{m}(\mathrm{p})
$$

gives

$$
\left\{\frac{\partial}{\partial r} A_{1}(\mathrm{p}, r)\right\}_{r=0}=\frac{1}{2} \frac{K_{0}^{2}-K_{1}^{2}}{\left(K_{0}+K_{1}\right)^{2}}
$$

and

$$
\begin{aligned}
& \left\{\frac{\partial^{2}}{\partial r^{2}} A_{1}(\mathrm{p}, r)\right\}_{r=0} \\
& =\frac{4 \mathrm{p}\left[K_{0}^{2}\left(8 \mathrm{p}^{2}+3 \mathrm{p}+24\right)-K_{1}^{2}\left(8 \mathrm{p}^{2}+3 \mathrm{p}+15\right)\right]-K_{0} K_{1}\left(15 \mathrm{p}^{4}-47 \mathrm{p}^{2}-192\right)}{24 \mathrm{p}^{2}\left(K_{0}+K_{1}\right)^{2}}
\end{aligned}
$$

where the argument, $\mathrm{p}$, of $K_{m}$ (p) has been omitted for convenience.

Hence, neglecting higher order than $r$, equations (51), (53), and (54) give

$$
\begin{aligned}
\bar{A}_{1}(\mathrm{p}, r) & =\left[1-\frac{K_{1}}{K_{0}+K_{1}}\right]+r\left[\frac{1}{2}-\frac{K_{1}}{K_{0}+K_{1}}\right]+\cdots \\
& =\left[1-\mathscr{L}\left(k_{1}(\mathrm{~J})\right)\right]+r\left[\frac{1}{2}-\mathscr{L}\left(k_{1}(\mathrm{~J})\right)\right]+\cdots
\end{aligned}
$$

where $k_{1}(\mathrm{~J})$ is Wagner's [4] "growth of lift" function. From (56) and (2)

$$
A_{1}(\mathrm{~J})=H(\mathrm{~J})\left[\left(1-k_{1}(\mathrm{~J})\right)+\pi \frac{c}{h}\left(\frac{1}{2}-k_{1}(\mathrm{~J})\right)+\cdots \cdot .\right.
$$

Thus, $A_{1}(\mathrm{~J})$ is the generalisation of Wagner's [4] "growth of lift" function. For small values of $r$, equations (39) and (57) may be used to determine $\lambda U$. 


\section{The Lift and Moment acting on an Aerofoil of the Cascade}

From Bernoulli's equation and the fact that $U$ is a function of $t$ only, we have, using equation (13) and the approximations consistent with linear perturbation theory, that the pressure distribution $p$ on an aerofoil surface is given by

$$
p=G(\mathrm{~J})+\rho U^{2} \Omega-\frac{2 x}{c} \rho U \frac{\partial U}{\partial \mathrm{J}}+\frac{2}{c} \rho U \int_{-c / 2}^{x} \frac{\partial}{\partial \mathrm{J}}(U \Omega) \mathrm{d} x .
$$

Following the procedure of Woods [8], this equation yields

$$
\begin{aligned}
p(\gamma, \mathrm{J}) & =G(\mathrm{~J})-2 \rho U \dot{U} \frac{x}{c}+\frac{\rho U^{2}}{2 \pi} \int_{-\pi}^{\pi} \theta\left(\gamma^{\prime}\right) \cot \frac{\gamma^{\prime}-\gamma}{2} \mathrm{~d} \gamma^{\prime} \\
& +\frac{\rho U}{2 \pi r} \int_{-\pi}^{\pi}(U \cdot \theta)\left(\int _ { 0 } ^ { \gamma } \left\{\frac{\sin \gamma^{*} \cot \left(\gamma^{\prime}-\gamma^{*}\right) / 2}{\cos \gamma^{*}+\cosh \mu}+1\right.\right. \\
& \left.\left.-\frac{\sinh ^{2} \mu}{\left(\cos \gamma^{*}+\cosh \mu\right)\left(\cos \gamma^{\prime}+\cosh \mu\right)}\right\} \mathrm{d} \gamma^{*}\right) \mathrm{d} \gamma^{\prime}
\end{aligned}
$$

Integration by parts and substitution for $\theta$ from (8), yields

$$
\begin{aligned}
p(\gamma, \mathrm{J})=p_{\mathrm{s}}(\gamma) & +2 \rho U U \frac{x}{c}-\frac{1}{2} \rho \lambda U^{2} \cot \frac{\gamma}{2} \\
& +\frac{\rho U}{2 \pi} \int_{-\pi}^{\pi}\left\{n\left(\gamma^{\prime}, \mathrm{J}\right)+\dot{N}\left(\gamma^{\prime}, \mathrm{J}\right)\right\} \cot \frac{\overline{\gamma^{\prime}-\gamma}}{2} \mathrm{~d} \gamma^{\prime}
\end{aligned}
$$

where

$$
N\left(\gamma^{\prime}, \mathrm{J}\right)=\frac{1}{r} \int_{0}^{\gamma^{\prime}} \frac{\left[n\left(\gamma^{*}, \mathrm{~J}\right)-\alpha U\right] \sin \gamma^{*}}{\cos \gamma^{*}+\cosh \mu} \mathrm{d} \gamma^{*}
$$

and $p_{\mathrm{B}}(\gamma)$ is the mean steady pressure.

Hence, we find that the lift, $L$, and the moment, $M$, about the chord mid-point are given by

$$
L=L_{\mathrm{s}}-\frac{c}{2 \gamma} \int_{-\pi}^{\pi} \frac{\left(p-p_{\mathrm{s}}\right) \sin \gamma}{\cos \gamma+\cosh \mu} \mathrm{d} \gamma
$$

and

$$
\begin{aligned}
M=M_{\mathrm{s}} & -\left(\frac{c}{2 r}\right)^{2} \int_{-\pi}^{\pi} \frac{\left(p-p_{\mathrm{s}}\right) \sin \gamma \ln (\cos \gamma+\cosh \mu)}{\cos \gamma+\cosh \mu} \mathrm{d} \gamma \\
& -\frac{c}{2 r}\left(L-L_{\mathrm{s}}\right) \ln \sinh \mu .
\end{aligned}
$$




\section{Accelerated Motion}

(I) General Case

Consider a particular rigid aerofoil of the cascade at a fixed incidence $\alpha$ and accelerating in an arbitrary fashion. Under these conditions

$$
n(\gamma, \mathrm{J})=0
$$

so that from (31) and (32)

$$
a_{0}=a_{1}=0 .
$$

Equations (39) and (41) then give, by (29), (30) and (38)

$$
\lambda U=2 \alpha\left[U-\frac{2}{\mathrm{e}^{r}+1} \int_{0}^{\mathrm{J}} U(\tau) A_{1}(\mathrm{~J}-\tau) \mathrm{d} \tau\right],
$$

and

$$
\chi=\frac{4 \alpha}{\mathrm{e}^{r}+1} \int_{0}^{\mathrm{J}} \dot{U}(\tau) A_{2}(\mathrm{~J}-\tau) \mathrm{d} \tau .
$$

Also, from (59), (60), (61) and (62)

$$
L=L_{\mathrm{s}}+\rho \frac{h U^{2} \lambda}{2}\left(1-\tanh \frac{\mu}{2}\right)-2 \rho \frac{h^{2} U U \alpha}{\pi c} \ln \left(1-\mathrm{e}^{-2 \mu}\right)
$$

and

(67) $\quad M=M_{\mathrm{s}}-\rho \frac{h^{2} U^{2} \lambda}{4 \pi}\left(1+\tanh \frac{\mu}{2}\right) \ln \left(1-\mathrm{e}^{-2 \mu}\right)-2 \rho \frac{h^{2} U \dot{U}}{\pi c} \ln \left(1-\mathrm{e}^{-\mu}\right)$.

If the aerofoil is suddenly given a velocity $U_{0}$, from rest, at $t=0$ then

$$
U(\mathrm{~J})=U_{0} H(\mathrm{~J})
$$

so that

$$
\dot{U}(\mathrm{~J})=U_{0} \delta(\mathrm{J})
$$

Hence, (64) and (66) give

$$
C_{L} \equiv \frac{L-L_{\mathrm{B}}}{\frac{1}{2} \rho U_{0}^{2}}=2 \alpha\left(\frac{h}{c}\right)\left(1-\tanh \frac{\mu}{2}\right)\left(1-\frac{2}{\mathrm{e}^{\mathrm{r}}+1} A_{1}(\mathrm{~J})\right)
$$

just after the start of the motion.

(II) Uniform acceleration

In the case of constant acceleration, we write

$$
U=u t
$$

where $u$ is a constant. Equation (13) then gives

$$
\mathrm{J}=\frac{u t^{2}}{c}
$$


so that

and

$$
U(\mathrm{~J})=\sqrt{c u \mathrm{~J}}
$$

$$
U(\mathrm{~J})=\sqrt{\frac{c u}{\mathrm{~J}}}=\frac{1}{2 \mathrm{~J}} U(\mathrm{~J})
$$

Equations (64) to (66) then become

$$
\begin{aligned}
\lambda U & =2 \alpha\left\{\sqrt{c u \mathrm{~J}}-\frac{\sqrt{c u}}{\mathrm{e}^{r}+1} \int_{0}^{\mathrm{J}} \tau^{-1 / 2} A_{1}(\mathrm{~J}-\tau) \mathrm{d} \tau\right\}, \\
\chi & =-\frac{2 \alpha \sqrt{c u}}{\mathrm{e}^{r}+1} \int_{0} \tau^{-1 / 2} A_{2}(\mathrm{~J}-\tau) \mathrm{d} \tau
\end{aligned}
$$

and

$$
L=L_{\mathrm{s}}+\rho \frac{h u \alpha c}{\pi}\left\{\pi \mathrm{J}-2\left(\frac{h}{c}\right) \ln \left(1-\mathrm{e}^{-2 \mu}\right)-\frac{\pi \mathrm{J}^{3 / 2}}{\mathrm{e}^{r}+1} \int_{0}^{\mathrm{J}} \tau^{-1 / 2} A_{1}(\mathrm{~J}-\tau) \mathrm{d} \tau\right\}
$$

\section{(III) Single Aerofoil}

The results for a single aerofoil are obtained by taking the limit $h \rightarrow \infty$. For example, from equations (57),

while equations (64) and (68) yield

$$
A_{1}(\mathrm{~J})=1-k_{1}(\mathrm{~J})
$$

$$
\lambda U=2 \alpha \int_{0}^{\mathrm{J}} U(\mathrm{~J}) k_{1}(\mathrm{~J}-\tau) \mathrm{d} \tau
$$

and

$$
C_{L}=2 \pi \alpha k_{1}(\mathrm{~J})
$$

These results are in agreement with the classical results for a single aerofoil (e.g. see [1]),

\section{References}

[1] Robinson, A. and Laurmann, J. A., Wing Theory, Cambridge Univ. Press (1956).

[2] Rosenblat, S., The aerodynamic forces on an aerofoil in non-uniform motion in a closed tunnel. Phil. Trans. Roy. Soc. A. 250, (1957) p. 247-278.

[3] Van der Pol, B. and Bremmer, H., Operational Calculus based on the Two-sided Laplace Integral. Cambridge Univ. Press. (1950)

[4] Wagner, H., Uber die Entstehung des dynamischen Auftriebes von Tragflügen. Z. angew. Math. Mech 5, (1925) p. 17-35.

[5] Watson, G. N., A Treatise on the Theory of Bessel Functions. (2nd Ed.) Cambridge Univ. Press (1952).

[6] Whittaker, E. T. and Watson, G. N. Modern Analysis. Cambridge Univ. Press. (1952).

[7] Woods, L. C., The two-dimensional subsonic flow of anunviscied fluid about an aerofoil of arbitrary shape. Rep. Memo. Aero. Res. Comm. London, No. 2811, (1953).

[8] Woods, L. C., An unsteady flow through a cascade of aerofoils Proc. Roy. Soc., A. 228, (1955) p. 50-65.

The University of New South Wales, Sydney. 